\title{
Keratoconus associated with cone-rod dystrophy
}

\author{
Balaji Kannan' \\ 'Senior Resident, Department Of Ophthalmology, Dhanalakshmi Srinivasan \\ Medical College \& Hospital, India
}

\begin{abstract}
Keratoconus is known to be associated with a variety of ocular and systemic disorders. The common posterior segment disorders known to be associated with keratoconus are retinitis pigmentosa, macular coloboma, Leber's congenital amaurosis, retinal aplasia and retrolental fibroplasias. ${ }^{1-3}$ Occurrences of keratoconus in association with tapetoretinal degeneration is rare and has been reported infrequently. ${ }^{4-5}$ Visualization of the fundus is often difficult in cases of keratoconus due to the associated refractive error and corneal opacities. This may make it difficult for the ophthalmologist to clinically diagnose associated macular degenerative changes preoperatively. We report a case of keratoconus who was diagnosed to have cone-rod dystrophy following successful corneal transplantation.
\end{abstract}

Key words: Keratoconus, bull's eye maculopathy, retinitis pigmentosa, electroretinogram

\section{Case report}

A 29-year-old man presented to the outpatient clinic with complaints of sudden painless decrease of vision, associated with watering in the right eye for the past one month. There was no history of any recent trauma. The patient was a known high myope since 15 years and a contact lens wearer for the last ten years. On examination, his best corrected visual acuity in the right eye was counting fingers at one meter and 20/200 in left eye. Slit lamp biomicroscopy of both eyes revealed features suggestive of advanced keratoconus, with acute hydrops in the both eye (right more than left eye). Fundus examination through the hazy cornea revealed pigmentary changes at the macula in both eyes.

Following medical management of the hydrops in the right eye, best corrected visual acuity with contact lens did not improve beyond 20/200. He underwent corneal transplantation in the right eye one year later. Postoperatively, despite a clear graft (Fig. 1), his best corrected visual acuity in the right eye did not improve beyond $20 / 120$. Keratometry readings in the right eye were $48.25 \times 128 \mathrm{deg} / 44.12 \mathrm{X}$ $38 \mathrm{deg}$. Fundus examination of both eyes revealed minimal temporal pallor of optic disc associated with pigmentary changes at the macula giving the appearance of bull's eye maculopathy (Fig. 2). An electroretinogram of both the eyes was done. Grossly delayed implicit time with reduced amplitudes of the rod response and extinguished cone waveforms were noted, indicating cone rod dystrophy (Fig. 3). Color blindness was also noted with the Ishihara's pseudoisochromatic color plates. Genetic counseling revealed it to be an isolated defect with negative family history.

Correspondence: Balaji Kannan.

E-mail: sdhrsnbalaji0@gmail.com 


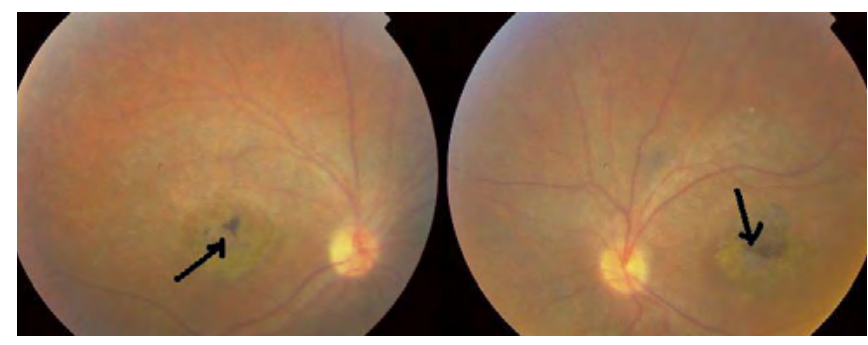

Fig. 1. Slit-lamp photograph of left eye showing healed acute hydrops (arrow) and clear corneal graft post PKP (right eye).

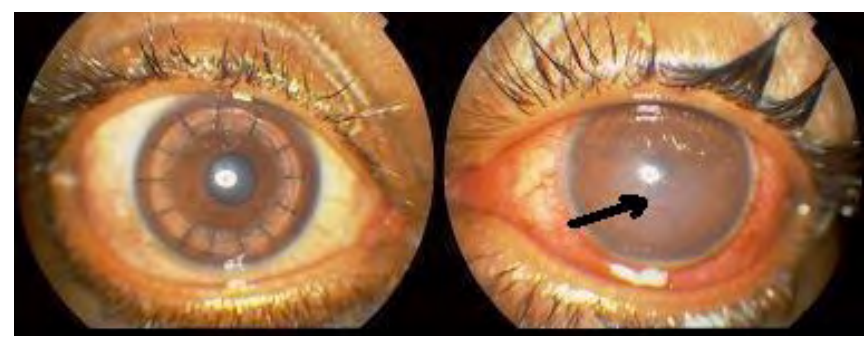

Fig. 2. Fundus photograph of the both eyes with Bull's eye maculopathy(arrow) and minimal temporal pallor.

\section{Discussion}

A correlation between keratoconus and tapetoretinal degeneration has been reported by Moschos and coworkers. ${ }^{4}$ Progressive cone dystrophy in association with keratoconus has also been reported in a 33 year old woman. ${ }^{5}$ Coexistence of these two conditions, especially photoreceptor dysfunction, has prognostic implications on the outcome of corneal transplantation in these cases. High refractive error and corneal opacities in keratoconus may often prevent visualization of the fundus. In our case a clear view of the fundus was not possible due to the corneal scarring which occurred following resolution of corneal hydrops. However, following corneal transplantation, it was possible to make a clinical diagnosis of bull's eye maculopathy, which was confirmed to be cone rod dystrophy on electro physiologic study.

Preoperative electro physiologic studies have been advised to evaluate retinal photoreceptor function, when a clear view of the fundus is not possible. Moschos and coworkers recommend avoiding corneal transplantation if electro physiologic studies show an abnormal response as it indicates photoreceptor dysfunction, usually associated with poor visual potential. ${ }^{4}$

Although corneal transplantation has not been advocated for patients of keratoconus with tapetoretinal degeneration, it may improve the quality of vision in these patients if not the absolute visual acuity, as was noted in our patient. After explaining the nature of disease and the visual outcome of corneal transplantation, our patient is now awaiting corneal transplantation in the left eye. Our case also strengthens the association between keratoconus and cone rod dystrophy. Hence it is essential to perform preoperative electro physiologic studies to evaluate the retinal activity and possible visual outcome, in patients with keratoconus associated with corneal opacity, which precludes a detailed fundus examination preoperatively. 

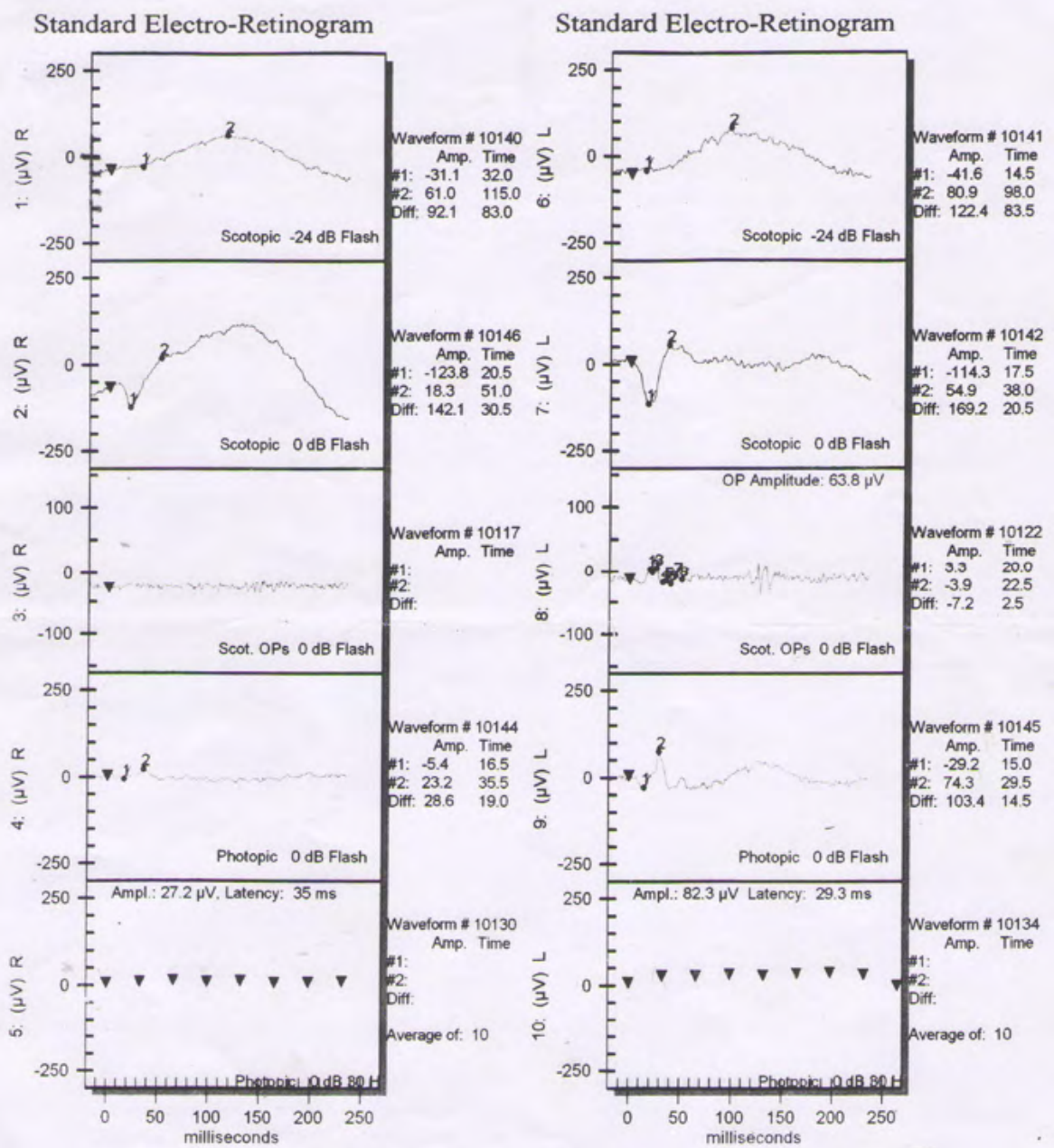

Fig. 3. ERG showing abnormal waveforms. Scotopic/blue flash-showing reduced amplitude and delayed implicit time in both eyes. Photopic/30 $\mathrm{Hz}$ showing extinguished waveforms in both eyes.

\section{References}

1. Freedman J, Gombos GM. Bilateral macular coloboma, keratoconus and retinitis pigmentosa. Ann Ophthalmol 1971;3:664-665.

2. Leighton DA, Harris R. Retinal aplasia in association with macular coloboma, keratoconus and cataract. Clin Genet 1973;4:270-274.

3. Heher KL, Traboulsi El, Maumenee IH. The natural history of Leber's congenital amaurosis. Age related findings in 35 patients. Ophthalmology 1992;99:241-245.

4. Moschos M, Droutsas D, Panagakis E, Tsioulias G, Tslouki M. Keratoconus and tapetoretinal degeneration. Cornea 1996;15:473-476.

5. Wilhelmus KR. Karatoconus and progressive cone dystrophy. Ophthalmologica 1995;209:278-279. 